\title{
Effect of Hall Current on MHD Oscillatory Slip Flow with Varying Temperature and Concentration
}

\author{
K. Sumathi ${ }^{1}$ T. Arunachalam ${ }^{2}$ and N. Radha ${ }^{1, *}$ \\ ${ }^{1}$ Department of Mathematics, PSGR Krishnammal College for Women, \\ Coimbatore 641004, Tamil Nadu, India \\ ${ }^{2}$ Department of Mathematics, Kumaraguru College of Technology, \\ Coimbatore 641049, Tamil Nadu, India \\ *Corresponding author's email: radha_nat2k5 [AT] yahoo.co.in
}

\begin{abstract}
An investigation of the effect of Hall current on an unsteady MHD mixed convective oscillatory flow of an electrically conducting fluid through a planar channel filled with saturated porous medium is carried out in this paper. The effect of buoyancy, heat source, thermal radiation, chemical reaction and Hall current are taken into account with slip velocity, varying temperature and concentration at the lower boundary. A series solution is found using perturbation techniques. The effects of various parameters on the main and cross flow velocity, temperature, Skin friction, rate of heat and mass transfer are discussed numerically.
\end{abstract}

Keywords--- Hall Current; Slip Flow; Oscillatory flow; Heat Source; Radiation

\section{INTRODUCTION}

The significance of MHD convection flows through porous media is far reaching in heat and mass transfer problems and has received rigorous attention in recent studies involving diverse fields like thermal generator, nuclear reactors, geothermal energy extractions and in the field of aerodynamics. Oscillatory flows simultaneously find their use in diverse engineering fields. In addition, Hall effects on the fluid flow with variable concentration play a major role in the field of power generators and accelerators in geophysics, in design of underground water energy storage system, soil sciences, astrophysics, nuclear power reactors and so on.

Ahmed et al [1] studied the unsteady MHD free convection flow past a vertical porous plate with thermal diffusion, heat source and Hall current. They showed that the decrease in the Soret effect leads to an increase in both main flow and cross flow velocity. Hall Effect is found to decrease the main flow velocity near the plate and increase the cross flow velocity.

Effects of radiation and Hall current on MHD flow of a viscous, Newtonian and electrically conducting fluid past a porous rotating infinite disk was investigated by Anjali Devi [2]. They showed that the increase in magnetic interaction lead to decrease in radial, tangential and axial velocities but results in increased temperature distribution while a decrease in the Hall parameter reduces the tangential velocity and temperature distribution. An investigation on Hall Effect with a chemically reacting flow for an oscillatory MHD convective, viscous, incompressible, radiating and electrically conducting fluid in a vertical porous rotating channel in slip flow regime was studied by Chand et al [3].

Hsiao [5] investigate the energy conversion problem for electric hydro magnetic heat and mass mixed convection in an incompressible second grade Maxwell fluid with radiation and viscous dissipation effects. Hsiao [6] analysed the numerical Soret- Dufour heat and mass transfer of a steady laminar boundary-layer flow with Ohmic dissipation, electromagnetic hydrodynamic, porosity and buoyancy multimedia physical features about extrusion processing for a stretching sheet.

Studies pertaining to oscillatory flow investigations in a planar channel with varying temperature and concentration field have recently been looked into the context of MHD slip flows with heat source by Raja Sekhar et al [7]. The study reveals that with an increase in magnetic field and a decrease in thermal Grashof number, a drop in velocity field is observed. However, such studies have never been performed in presence of a magnetic field wherein the influence of Hall effects may also come into prominence. 
Later on, Rakesh Kumar [8] studied the unsteady MHD free convection flow of viscoelastic fluid past an infinite vertical porous plate with slip through a porous medium with oscillating temperature and Hall currents taken into consideration. This study showed that primary velocity decreases with increase in Hall current but Grashof number has a reverse effect for the secondary velocity profiles.

\section{*Corresponding Author}

Singh and Kumar [9] studied the periodic solution of an oscillatory MHD flow of an electrically conducting fluid with Hall current through two horizontal porous plates subjected to a perpendicular magnetic field. They found that the resultant velocities rapidly increase from the stationary plate and approach unity in the form of a damped oscillation while the resultant shear stress goes on increasing with increasing rotation of the channel.

On the other hand chemically reacting unsteady MHD oscillatory slip flows in a planar channel with varying concentration, thermal radiation, heat source and magnetic field effect was studied by Sivaraj and Rushi [10]. Velocity profiles accelerate for increasing value of Grashof number and permeability of the porous medium whereas velocity profiles decrease with increase in the magnetic field and frequency of oscillation parameter.

In this paper, the work of Raja Sekhar et al [7] has been extended to study the effect of Hall current on unsteady MHD flow of an electrically conducting fluid through planar porous plates in the presence of a magnetic field. Approximate solutions for the velocity, temperature and concentration field are obtained, thereby estimating the Skin friction, Nusselt number and Sherwood number values. The influence of the fluid flow parameters on the heat and mass transfer characteristics are studied and discussed along with graphical representation.

\section{FLOW DESCRIPTION AND GOVERNING EQUATIONS}

We consider the two dimensional unsteady oscillatory slip flow of an electrically conducting Newtonian fluid confined between two vertical plates. The plates are porous in nature, wherein the left plate is associated with a slip parameter. A magnetic field which is strong enough to produce Hall current is applied perpendicular to the fluid flow. We have introduced a Cartesian coordinate system, $x^{*}$ - axis is taken along the flow and the $y^{*}-$ axis is taken perpendicular to the flow starting from the left plate.

The equation of continuity $\nabla \cdot \vec{V}=0$ on integration gives $v^{*}=v_{0}$, where $u^{*}, v^{*}$ and $w^{*}$ are the components of velocity in $x^{*}, y^{*}$ and $z^{*}$ directions respectively. $\vec{J}=\left(J_{x}^{*}, J_{y}^{*}, J_{z}^{*}\right)$ are the components of electric current density $\vec{J}$. The equation of conservation of electric charge, $\nabla . \vec{J}=0$, gives $J_{y}^{*}=$ constant. Since the plates are electrically nonconducting $J_{y}^{*}=0$ and is zero everywhere in the flow.

Considering Hall Effect in generalized Ohms law we get the equation as below

$$
\vec{J}+\frac{\omega_{e} \tau_{e}}{B_{0}}(\vec{J} \times \vec{B})=\sigma\left(\vec{E}+\vec{V} \times \vec{B}+\frac{1}{e \eta_{e}} \nabla p_{e}\right)
$$

where $\vec{V}, \sigma, \omega_{e}, \tau_{e}, e, \eta_{e}$ and $p_{e}$ denote the velocity, electric conductivity, magnetic permeability, cyclotron frequency of the electrons, electron collision time, electric charge, number of density of electron and pressure electron respectively.

In the absence of an external electric field $\vec{E}=0$, Ohms' law reduces to the form

$$
\vec{J}+\frac{m}{B_{0}}(\vec{J} \times \vec{B})=\sigma\left(\vec{V} \times \vec{B}+\frac{1}{e \eta_{e}} \nabla p_{e}\right)
$$

where $m=\omega_{e} \tau_{e}$ is the Hall parameter.

When the strength of the magnetic field is very large,

$$
\begin{aligned}
& j_{x}^{*}-\omega_{e} \tau_{e} j_{z}^{*}=\sigma B_{o} w^{*} \\
& j_{z}^{*}+\omega_{e} \tau_{e} j_{x}^{*}=-\sigma B_{o} u^{*}
\end{aligned}
$$


The solution of equations (3) and (4) are

$$
\begin{aligned}
& j_{x}^{*}=\frac{\sigma B_{0}\left(m u^{*}-w^{*}\right)}{\left(1+m^{2}\right)} \\
& j_{z}^{*}=\frac{\sigma B_{0}\left(u^{*}+m w^{*}\right)}{\left(1+m^{2}\right)}
\end{aligned}
$$

Further, taking into account Boussinesq approximation, Heat source and Radiation effects in the incompressible, viscous flow, the governing equations for the momentum, energy and concentration are given as follows

$$
\begin{array}{lll}
\frac{\partial u^{*}}{\partial t^{*}}+v^{*} \frac{\partial u^{*}}{\partial y^{*}} & = & g \beta\left(T^{*}-T_{0}^{*}\right)+g \beta\left(C^{*}-C_{0}^{*}\right)+v\left(\frac{\partial^{2} u^{*}}{\partial y^{* 2}}\right)-\frac{v}{K} u^{*}-\frac{\sigma B_{0}^{2}\left(u^{*}+m w^{*}\right)}{\rho\left(1+m^{2}\right)} \\
\frac{\partial w^{*}}{\partial t^{*}}+v^{*} \frac{\partial w^{*}}{\partial y^{*}} & = & v\left(\frac{\partial^{2} w^{*}}{\partial y^{* 2}}\right)-\frac{v}{K} w^{*}+\frac{\sigma B_{0}^{2}\left(m u^{*}-w^{*}\right)}{\rho\left(1+m^{2}\right)} \\
\frac{\partial T^{*}}{\partial t^{*}}+v^{*} \frac{\partial T^{*}}{\partial y^{*}} & = & \frac{\kappa}{\rho C_{p}}\left(\frac{\partial^{2} T^{*}}{\partial y^{* 2}}\right)-\frac{1}{\rho C_{p}} \frac{\partial q_{r}}{\partial y}+\frac{Q^{*}}{\rho C_{p}}\left(T^{*}-T_{0}^{*}\right) \\
\frac{\partial C^{*}}{\partial t^{*}}+v^{*} \frac{\partial C^{*}}{\partial y^{*}} & = & D\left(\frac{\partial^{2} C^{*}}{\partial y^{* 2}}\right)
\end{array}
$$

where $v$ is kinematic viscosity, $\kappa$ is thermal conductivity, $C_{\mathrm{p}}$ is specific heat at constant pressure, $\vec{g}$ is acceleration due to gravity, $\sigma$ is the electrical conductivity, $B_{0}$ is magnetic flux density, $T^{*}$ is the temperature of the fluid, $Q^{*}$ is the coefficient of heat source, $C^{*}$ is the concentration of the fluid, $K^{*}$ is permeability of the porous medium, $m$ is the Maxwell's reflection coefficient and $D$ is chemical molecular diffusivity.

The corresponding boundary conditions are

$$
\begin{aligned}
& u^{*}=L^{*} \frac{\partial u^{*}}{\partial y^{*}} ; \quad w^{*}=L^{*} \frac{\partial w^{*}}{\partial y^{*}} ; \quad T^{*}=T_{0}^{*}+\delta_{T} \frac{\partial T^{*}}{\partial y^{*}} ; \quad C^{*}=C_{0}^{*}+\delta_{c} \frac{\partial C^{*}}{\partial y^{*}} \quad \text { at } y^{*}=0 \\
& u^{*}=0 ; \quad w^{*}=0 ; \quad T^{*}=T_{d}^{*}+\delta_{T} \frac{\partial T^{*}}{\partial y^{*}} ; \quad C^{*}=C_{d}^{*}+\delta_{c} \frac{\partial C^{*}}{\partial y^{*}} \quad \text { at } y^{*}=d
\end{aligned}
$$

where $L^{*}=\left(\frac{2-m}{m}\right) L, L=\mu\left(\frac{\pi}{2 P \rho}\right)^{1 / 2}$ is the mean free path.

Cogley et al [4] have shown that, for the optically thin fluid flow, radioactive heat flux is represented by the following form:

$$
\frac{\partial q_{r}^{*}}{\partial y^{*}}=4\left(T^{*}-T_{\infty}^{*}\right) I^{*}
$$

where $I^{*}=\int K_{\lambda w} \frac{\partial e_{b \lambda}}{\partial T^{*}} d \lambda$

is the absorption coefficient at the plate and $e_{b \lambda}$ is plank constant.

By introducing the following non-dimensional parameters

$$
\begin{aligned}
& y=\frac{y^{*}}{d} ; \quad z=\frac{z^{*}}{d} ; \quad t=\frac{u_{0} t^{*}}{d} ; \quad p=\rho u_{0}^{2} p^{*} ; \quad u=\frac{u^{*}}{u_{0}} ; \quad v=\frac{v^{*}}{u_{0}} \text {; } \\
& w=\frac{w^{*}}{u_{0}} ; \quad d_{1}=\frac{\delta_{T}^{*}}{d} ; \quad d_{2}=\frac{\delta_{C}^{*}}{d} ; \quad \theta=\frac{T^{*}-T_{0}^{*}}{T_{d}^{*}-T_{0}^{*}} ; \quad C=\frac{C^{*}-C_{0}^{*}}{C_{d}^{*}-C_{0}^{*}} ; \\
& \operatorname{Re}=\frac{u_{0} d}{v} \text {, Reynolds number; } \quad S c=\frac{v}{D} \text {, Schmidt number; }
\end{aligned}
$$


$Q=\frac{Q^{*} v}{\rho C_{p} u_{0}^{2}}$, Heat Source;

$M=\frac{\sigma B_{0}{ }^{2} d^{2}}{\rho v}$, Hartmann Number;

$G m=\frac{g \beta v\left(C_{d}^{*}-C_{0}^{*}\right)}{u_{0}^{3}}$, Modified Grashof Number; $\quad F=\frac{4 v I}{\rho C_{p} u_{0}^{2}}$, Radiation Parameter;

$h=\frac{L^{*}}{d}$, Slip parameter due to main flow velocity; $S=\frac{V_{0}}{u_{0}}$, Suction Parameter;

The governing equations (7) - (10) can be written in non-dimensional form as

$$
\begin{array}{llll}
\operatorname{Re} \frac{\partial u}{\partial t}+\operatorname{Re} S \frac{\partial u}{\partial y} & = & G r \operatorname{Re}^{2} \theta+G m \operatorname{Re}^{2} C+\frac{\partial^{2} u}{\partial y^{2}}-\frac{1}{K} u-\frac{M(u+m w)}{\left(1+m^{2}\right)} \\
\operatorname{Re} \frac{\partial w}{\partial t}+\operatorname{Re} S \frac{\partial w}{\partial y} & = & \frac{\partial^{2} w}{\partial y^{2}}-\frac{1}{K} w+\frac{M(m u-w)}{\left(1+m^{2}\right)} \\
\frac{\partial \theta}{\partial t}+S \frac{\partial \theta}{\partial y} & = & \frac{1}{\operatorname{Pr} \operatorname{Re}} \frac{\partial^{2} \theta}{\partial y^{2}}+\operatorname{Re}(Q-F) \theta \\
\frac{\partial C}{\partial t}+S \frac{\partial C}{\partial y} & = & \frac{1}{S c \operatorname{Re}} \frac{\partial^{2} C}{\partial y^{2}}
\end{array}
$$

The corresponding boundary conditions in non-dimensional form are

$$
\begin{aligned}
& u=h \frac{\partial u}{\partial y} ; \quad w=h \frac{\partial w}{\partial y} ; \quad \theta=d_{1} \frac{\partial \theta}{\partial y} ; \quad C=d_{2} \frac{\partial C}{\partial y} \quad \text { at } y=0 \\
& u=0 ; \quad \quad w=0 ; \quad \theta=1+d_{1} \frac{\partial \theta}{\partial y} ; \quad C=1+d_{2} \frac{\partial C}{\partial y} \quad \text { at } y=1
\end{aligned}
$$

Here $\operatorname{Pr}, S, \operatorname{Re}$ denote Prandtl number, Suction parameter and Reynolds number respectively.

\section{SOLUTION OF THE PROBLEM}

Introducing $q=u(y, t)+i w(y, t)$ and $i=\sqrt{-1}$, the equations (15) and (16) transform to

$\operatorname{Re} \frac{\partial q}{\partial t}+\operatorname{Re} S \frac{\partial q}{\partial y} \quad=\quad G r \operatorname{Re}^{2} \theta+G m \operatorname{Re}^{2} C-\frac{q}{K}+\frac{\partial^{2} q}{\partial y^{2}}-\frac{M(1-i m) q}{\left(1+m^{2}\right)}$

subject to the boundary conditions

$$
\begin{array}{llll}
q=h \frac{\partial q}{\partial y} ; & \theta=d_{1} \frac{\partial \theta}{\partial y} ; & C=d_{2} \frac{\partial C}{\partial y} & \text { at } y=0 \\
q=0 ; & \theta=1+d_{1} \frac{\partial \theta}{\partial y} ; & C=1+d_{2} \frac{\partial C}{\partial y} & \text { at } y=1
\end{array}
$$

In order to solve equations (17), (18) and (20) with respect to boundary condition (21), we assume

$$
\begin{aligned}
& q(y, t)=q_{0}(y) e^{i \delta t} \\
& \theta(y, t)=\theta_{0}(y) e^{i \delta t} \\
& C(y, t)=C_{0}(y) e^{i \delta t}
\end{aligned}
$$

Now using (22) in equations (17), (18) and (20) we get

$$
\begin{array}{lll}
q_{0}^{\prime \prime}-S \operatorname{Re} q_{0}^{\prime}-N q_{0} & = & -G r \operatorname{Re}^{2} \theta-G m \operatorname{Re}^{2} C \\
\theta_{0}^{\prime \prime}-S \operatorname{Pr} \operatorname{Re} \theta_{0}^{\prime}+\operatorname{Pr} \operatorname{Re}(\operatorname{Re}(Q-F)-i \delta) \theta & = & 0 \\
C_{0}^{\prime \prime}-S \operatorname{Re} S c C_{0}^{\prime}-i \delta C_{0} & = & 0
\end{array}
$$


where $N=\frac{M}{1+m^{2}}(1-i m)+\frac{1}{k}+\operatorname{Re} i \delta$

Boundary conditions are
$q_{0}=h \frac{\partial q_{0}}{\partial y}$;
$\theta_{0}=d_{1} \frac{\partial \theta_{0}}{\partial y}$
$C_{0}=d_{2} \frac{\partial C_{0}}{\partial y}$
at $y=0$
$q_{0}=0$;
$\theta_{0}=1+d_{1} \frac{\partial \theta_{0}}{\partial y}$
$C_{0}=1+d_{2} \frac{\partial C_{0}}{\partial y}$
at $y=1$

The solutions of the equations (23)-(25) under the boundary conditions (26) are

$$
\begin{array}{rll}
q(y, t) & = & \left(A_{5} e^{m_{5} y}+A_{6} e^{m_{6} y}+A_{7} e^{m_{1} y}+A_{8} e^{m_{2} y}+A_{9} e^{m_{3} y}+A_{10} e^{m_{4} y}\right) e^{i \delta t} \\
\theta(y, t) & = & \left(A_{1} e^{m_{1} y}+A_{2} e^{m_{2} y}\right) e^{i \delta t} \\
C(y, t) & = & \left(A_{3} e^{m_{3} y}+A_{4} e^{m_{4} y}\right) e^{i \delta t}
\end{array}
$$

where $m_{1}=\frac{S \operatorname{Pr} \operatorname{Re}+\sqrt{S^{2} \operatorname{Pr}^{2} \operatorname{Re}^{2}-4 \operatorname{Pr} \operatorname{Re}(\operatorname{Re}(Q-F)-i \delta)}}{2} m_{2}=\frac{S \operatorname{Pr} \operatorname{Re}-\sqrt{S^{2} \operatorname{Pr}^{2} \operatorname{Re}^{2}-4 \operatorname{Pr} \operatorname{Re}(\operatorname{Re}(Q-F)-i \delta)}}{2}$

$$
\begin{array}{ll}
m_{3}=\frac{S c \operatorname{Re} S+\sqrt{(S)^{2}(S c)^{2} \operatorname{Re}^{2}+4 i \delta}}{2} ; & m_{4}=\frac{S c \operatorname{Re} S-\sqrt{(S)^{2}(S c)^{2} \operatorname{Re}^{2}+4 i \delta}}{2} ; \\
m_{5}=\frac{S \operatorname{Re}+\sqrt{S^{2} \operatorname{Re}^{2}+4 N}}{2} ; & m_{6}=\frac{S \operatorname{Re}-\sqrt{S^{2} \operatorname{Re}^{2}+4 N}}{2} ; \\
A_{1}=\frac{d_{1} m_{2}-1}{\left(1-d_{1} m_{1}\right)\left(1-d_{1} m_{2}\right)\left(e^{m_{2}}-e^{m_{1}}\right)} ; & A_{2}=\frac{1-d_{1} m_{1}}{\left(1-d_{1} m_{1}\right)\left(1-d_{1} m_{2}\right)\left(e^{m_{2}}-e^{m_{1}}\right)} ; \\
A_{3}=\frac{d_{2} m_{4}-1}{\left(1-d_{2} m_{3}\right)\left(1-d_{2} m_{4}\right)\left(e^{m_{4}}-e^{m_{3}}\right)} ; & A_{4}=\frac{1-d_{2} m_{3}}{\left(1-d_{2} m_{3}\right)\left(1-d_{2} m_{4}\right)\left(e^{m_{4}}-e^{m_{3}}\right)} \\
A_{5}=\frac{A_{11} e^{m_{6}}-\left(1-h m_{6}\right) A_{12}}{\left(1-h m_{5}\right) e^{m_{6}}-\left(1-h m_{6}\right) e^{m_{5}}} ; & A_{6}=\frac{A_{12}\left(1-h m_{5}\right)-A_{11} e^{m_{5}}}{\left(1-h m_{5}\right) e^{m_{6}}-\left(1-h m_{6}\right) e^{m_{5}}} ; \\
A_{7}=\frac{-G r \operatorname{Re}^{2} A_{1}}{m_{1}^{2}-S \operatorname{Re} m_{1}-N} ; & A_{8}=\frac{-G r \operatorname{Re}^{2} A_{2}}{m_{2}^{2}-S \operatorname{Re}_{2}-N} ; \\
A_{9}=\frac{-G m \operatorname{Re}^{2} A_{3}}{m_{3}^{2}-S \operatorname{Re} m_{3}-N} ; & A_{10}=\frac{-G m \operatorname{Re}^{2} A_{4}}{m_{4}^{2}-S \operatorname{Re}_{4}-N} ; \\
A_{11}=A_{7}\left(m_{1} h-1\right)+A_{8}\left(m_{2} h-1\right)+A_{9}\left(m_{3} h-1\right)+A_{10}\left(m_{4} h-1\right) ; & A_{12}=-\left(A_{7} e^{m_{1}}+A_{8} e^{m_{2}}+A_{9} e^{m_{3}}+A_{10} e^{m_{4}}\right) ;
\end{array}
$$

\section{SKIN FRICTION, HEAT AND MASS FLUX}

The Skin friction at the wall is given by

$$
\begin{aligned}
& \tau=\left(\frac{d q}{d y}\right)_{y=0}=\left(m_{5} A_{5}+m_{6} A_{6}+m_{1} A_{7}+m_{2} A_{8}+m_{3} A_{9}+m_{4} A_{10}\right) \\
& \tau=\left(\frac{d q}{d y}\right)_{y=1}=\left(\begin{array}{l}
m_{5} A_{5} e^{m_{5}}+m_{6} A_{6} e^{m_{6}}+m_{1} A_{7} e^{m_{1}} \\
+m_{2} A_{8} e^{m_{2}}+m_{3} A_{9} e^{m_{3}}+m_{4} A_{10} e^{m_{4}}
\end{array}\right)
\end{aligned}
$$

The heat flux in terms of Nusselt Number is given by

$$
\begin{aligned}
& N u=\left(\frac{d \theta}{d y}\right)_{y=0}=\left(m_{1} A_{1}+m_{2} A_{2}\right) \\
& N u=\left(\frac{d \theta}{d y}\right)_{y=1}=\left(m_{1} A_{1} e^{m_{1}}+m_{2} A_{2} e^{m_{2}}\right)
\end{aligned}
$$

The mass flux in terms of Sherwood Number is given by 


$$
\begin{aligned}
& S h=\left(\frac{d C}{d y}\right)_{y=0}=\left(m_{3} A_{3}+m_{4} A_{4}\right) \\
& S h=\left(\frac{d C}{d y}\right)_{y=1}=\quad\left(m_{3} A_{3} e^{m_{3}}+m_{4} A_{4} e^{m_{4}}\right)
\end{aligned}
$$

\section{NUMERICAL RESULTS}

In this paper, effect of Hall current on unsteady, MHD mixed convective oscillatory flow of an electrically conducting optically thin fluid flow through a planar channel filled with saturated porous medium was investigated. Analytical expressions obtained for velocity, temperature and concentration were evaluated numerically and the results are illustrated graphically in figures (1) - (27).

Figures (1) - (6) show that there is a drop in the velocity field due to increase in Prandtl number, Heat source parameter, Hartmann number, Slip parameter and Reynolds number. The velocity field is found to increase due to increase in Hall parameter. Increase in Permeability parameter accelerates the flow velocity due to increase in pore space.

From Figures (7) and (8), the magnitude of the cross flow velocity is found to be increasing due to increase in Reynolds number. When the Hall parameter is small, the cross flow velocity increases with increase in Hall parameter. When the Hall parameter is large, increase in Hall parameter tend to decelerate the cross flow velocity.

Variations in the temperature profile due to various parameters are plotted through figures (9) - (12). From these figures it may be inferred that temperature profile gets decreased due to increase in Heat source parameter. Increasing Prandtl number decreases the temperature distribution which is in agreement with the behaviour observed by RajaSekhar et al [5]. Temperature distribution is enhanced due to increase in Suction parameter and volumetric temperature expansion.

Figures (13) - (15) portray that the concentration profile increase due to increase in Hall parameter, Suction parameter, Schmidt number and volumetric concentration expansion. Figures (16) - (19) illustrate that Skin friction increases with increase in Hall parameter and Heat source parameter decreases the wall shear stress at $y=0$. Skin friction decreases with increasing Hall parameter and increases with increasing heat source parameter.

The dimensionless rate of heat transfer is shown in Figures (20) - (23). At both the plates due to increase in Heat source parameter, the heat flux is found to be decreasing and the increase in Suction parameter increase the heat flux at both the plates.

It is evident from figures (24) - (27), the mass flux enhances due to increase in Schmidt number and Suction parameter at the left plate. The rate of mass transfer decreases due to increase in Schmidt number and Suction parameter at $y=1$.

\section{CONCLUSION}

In this paper, we have extended the work of RajaSekhar et al [7] to study the effect of Hall current on unsteady, MHD mixed convective oscillatory flow of an electrically conducting optically thin fluid flow through a planar channel filled with saturated porous medium. Analytical expressions obtained for velocity, temperature and concentration were evaluated numerically.

The main findings of this study are

$>$ There is a drop in the velocity field due to increase in Prandtl number, Heat source parameter, Hartmann number, slip parameter and Reynolds number. The velocity field is found to increase due to increase in Hall parameter.

$>$ Increase in Permeability parameter accelerates the flow velocity due to increase in pore space.

$>$ When the Hall parameter is small, the cross flow velocity increases with increase in Hall parameter. When the Hall parameter is large, increase in Hall parameter tend to decelerate the cross flow velocity.

$>$ Temperature profile gets decreased due to increase in Heat source parameter. Increasing Prandtl number decreases the temperature distribution.

$>$ Temperature distribution is enhanced due to increase in Suction parameter and volumetric temperature expansion.

$>$ At both the plates due to increase in Heat source parameter, the heat flux is found to be decreasing and the increase in Suction parameter increase the heat flux at both the plate.

$>$ The rate of mass transfer decreases due to increase in Schmidt number and Suction parameter at $y=1$. 
Mass flux enhances due to increase in Schmidt number and Suction parameter at the left plate.

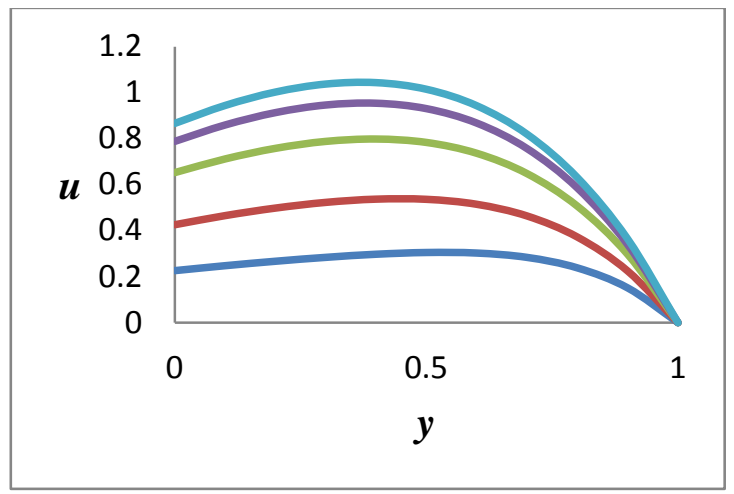

Figure 1: Main Velocity u versus y for $\operatorname{Re}=1, S c=0.22, S=1$, $\operatorname{Pr}=0.71, t=1, \delta=0.5 M=10, G r=2, G m=1, Q=1, F=1$ $d_{1}=0.5, d_{2}=0.5, h=1, K=2 . m=1,3,5,7,9$

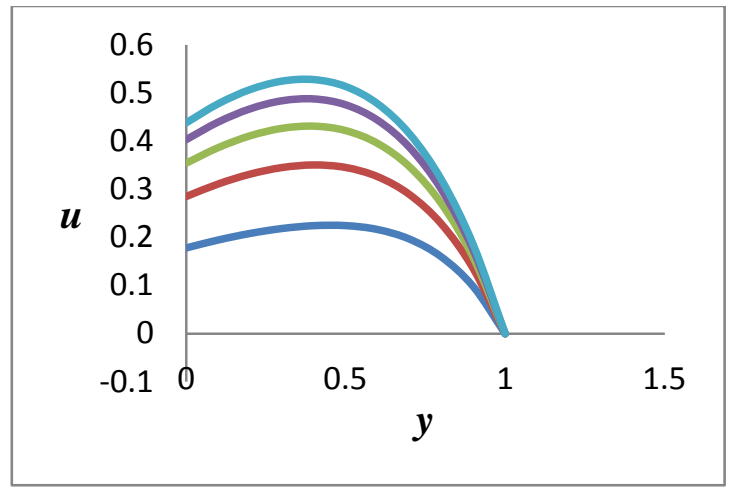

Figure 3: Main Velocity u versus y for $\operatorname{Re}=1, S c=0.22, S=1$, $\operatorname{Pr}=0.71, t=1, \delta=0.5 M=10, G r=2, G m=1, Q=1, F=1$, $d_{1}=0.5, d_{2}=0.5, h=1, m=10 . K=0.1,0.2,0.3,0.4,0.5$

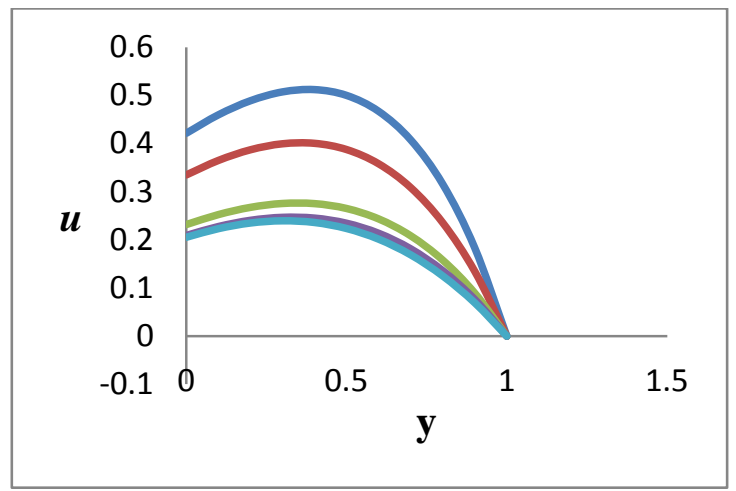

Figure 5: Main Velocity u versus y for $\operatorname{Re}=1, S c=0.22 S=1$, $\operatorname{Pr}=0.71, t=1, \delta=0.5 M=10, G r=2, G m=1, m=10$, $F=1, d_{1}=0.5, d_{2}=0.5, h=1, K=2 ., Q=1,3,5,7,9$

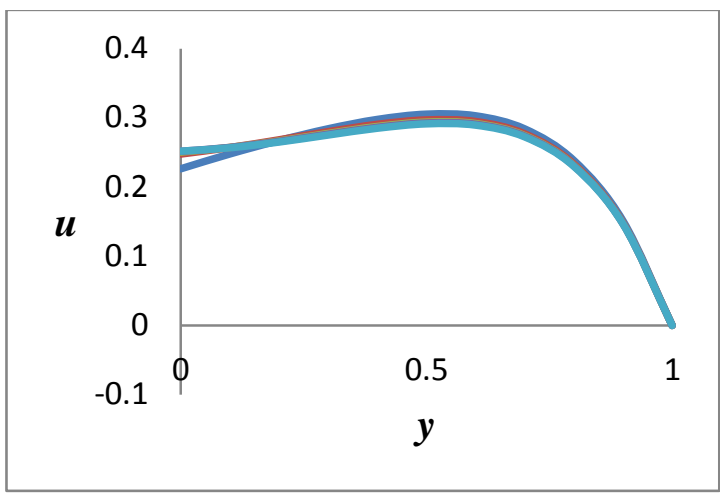

Figure 2: Main Velocity u versus y for $\mathrm{Re}=1, S c=0.22, S=1$, $\operatorname{Pr}=0.71, t=1, \delta=0.5 M=10, G r=2, G m=1, Q=1, F=1$, $d_{1}=0.5, d_{2}=0.5, m=10, K=2 ., h=1,3,5,7,9$

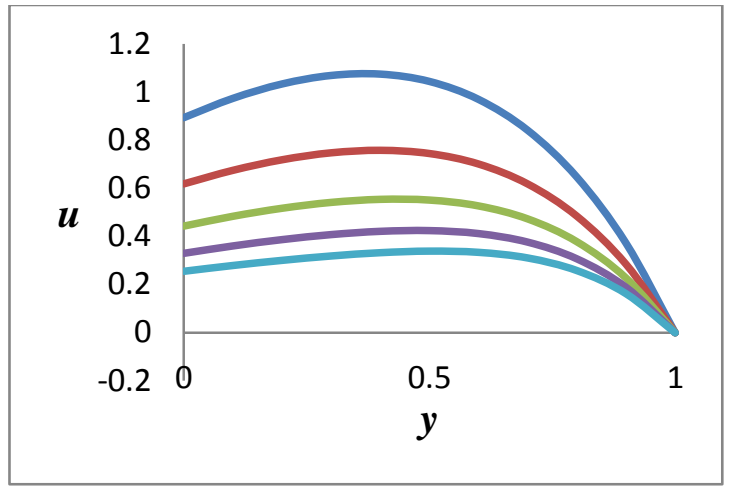

Figure 4: Main Velocity u versus y for $\operatorname{Re}=1, S c=0.22, S=1$, $\operatorname{Pr}=0.71, t=1, \delta=0.5 m=10, G r=2, G m=1, Q=1, F=1$, $d_{1}=0.5, d_{2}=0.5, h=1, K=2 ., M=1,3,5,7,9$

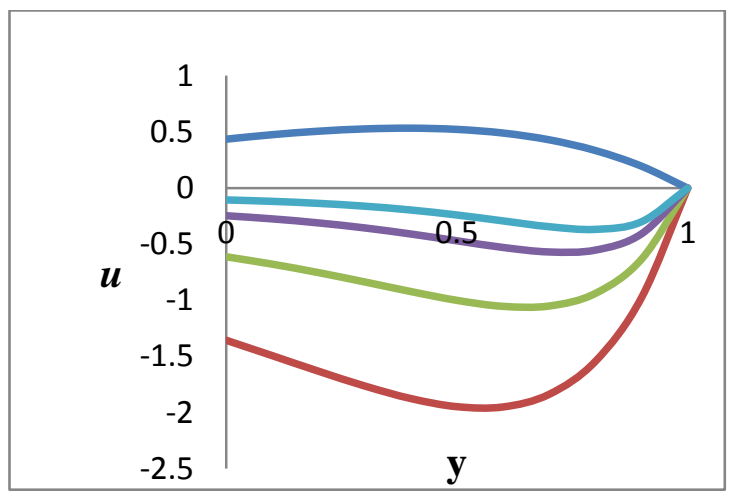

Figure 6: Main Velocity u versus y for $m=10, S c=0.22, S=1$, $\operatorname{Pr}=0.71, t=1, \delta=0.5 M=10, G r=2, G m=1, Q=1, F=1$ $d_{1}=0.5, d_{2}=0.5, h=1, K=2 ., \operatorname{Re}=1,2,3,4,5$ 


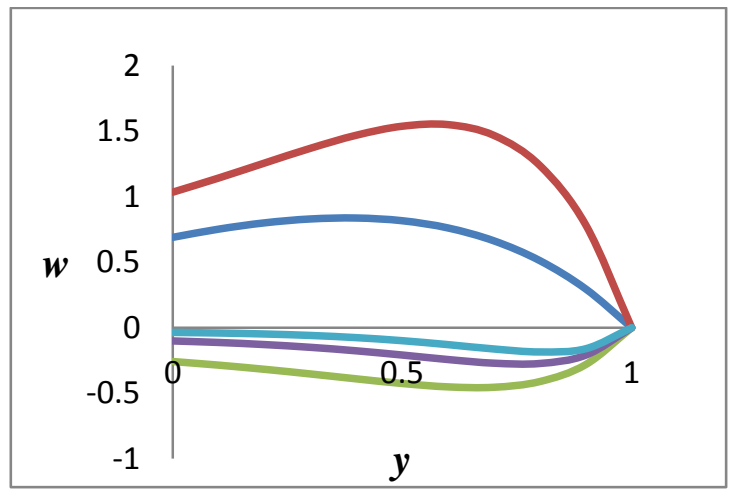

Figure 7: Cross Velocity w versus y for $m=10, S c=0.22, S=1$, $\operatorname{Pr}=0.71, t=1, \delta=0.5, M=10, G r=2, G m=1, Q=1, F=1$ $d_{1}=0.5, d_{2}=0.5, h=1, K=2 ., \operatorname{Re}=1,2,3,4,5$

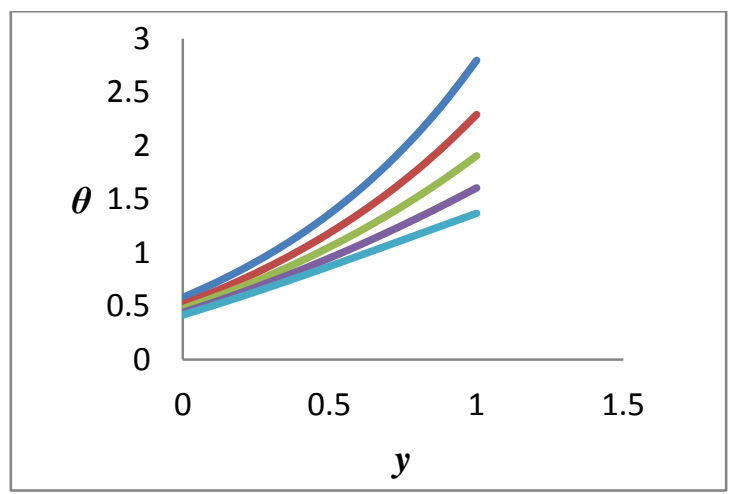

Figure 9: Temperature $\theta$ versus y for $\operatorname{Re}=1, S c=0.22, S=1$, $\operatorname{Pr}=0.71, t=1, \delta=0.5 \quad M=10, G r=2, G m=1, m=10$, $F=1, d_{1}=0.5, d_{2}=0.5, h=1, K=2 ., Q=0.5,1.0,1.5,2.0,2.5$

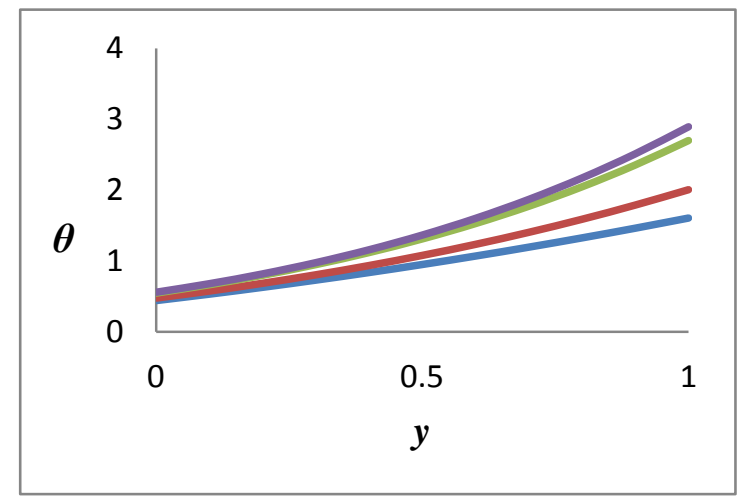

Figure 11: Temperature $\theta$ versus y for $\operatorname{Re}=1, S c=0.22, m=10$, $\operatorname{Pr}=0.71, t=1, \delta=0.5, M=10, G r=2, G m=1, Q=1, F=1$ $d_{1}=0.5, d_{2}=0.5, h=1, K=2 ., S=1.0,1.5,2.0,2.5$

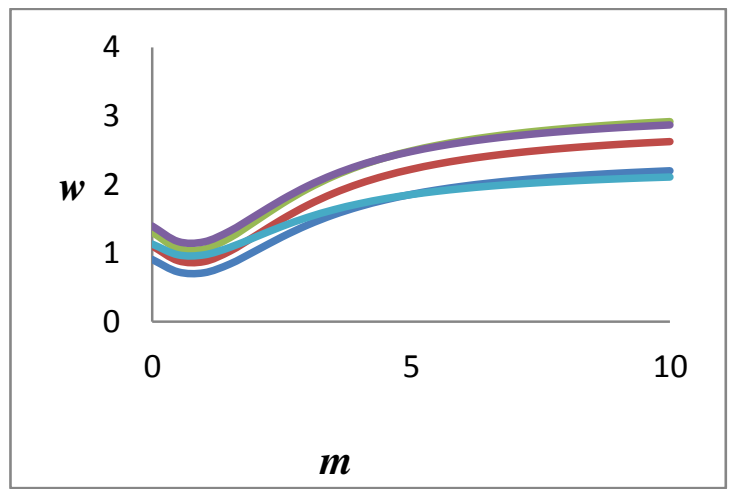

Figure 8: Cross Velocity w versus $\mathrm{m}$ for $\mathrm{Re}=1, S c=0.22, S=1$, $\operatorname{Pr}=0.71, t=1, \delta=0.5, M=10, G r=2, G m=1, Q=1, F=1$ $d_{1}=0.5, d_{2}=0.5, h=1, K=2 ., y=0,0.2,0.4,0.6,0.8$

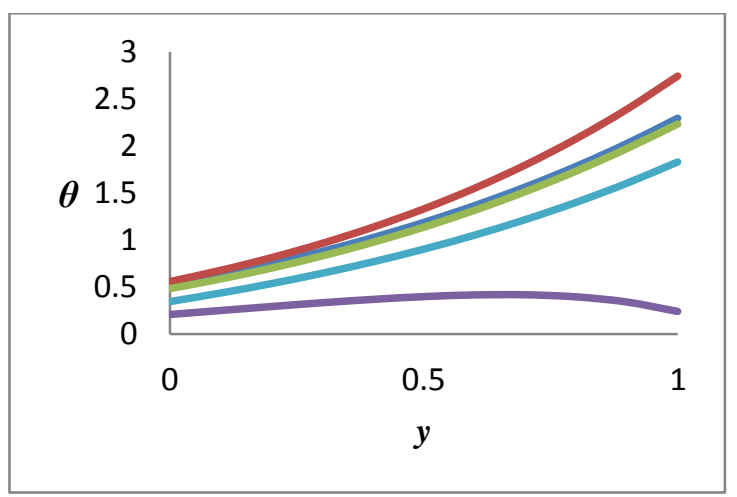

Figure 10: Temperature $\theta$ versus y for $\operatorname{Re}=1, S c=0.22, S=1$, $m=10, t=1, \delta=0.5, M=10, G r=2, G m=1, Q=1, F=1$ $d_{1}=0.5, d_{2}=0.5, h=1, K=2 ., \operatorname{Pr}=0.71,1.0,1.5,2.0,2.5$

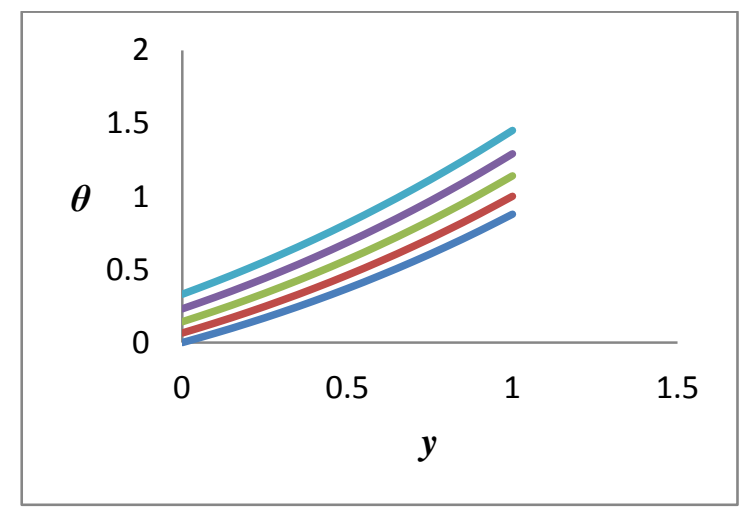

Figure 12: Temperature $\theta$ versus y for $\operatorname{Re}=1, S c=0.22, S=1$, $\operatorname{Pr}=0.71, t=1, \delta=0.5, M=10, G r=2, G m=1, Q=1, F=1$ $m=10, d_{2}=0.5, h=1, K=2 ., d 1=0,0.1,0.2,0.3,0.4$ 


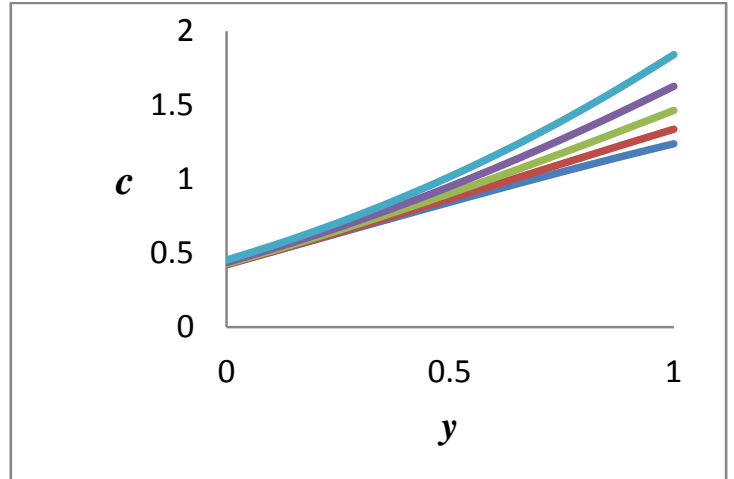

Figure 13: Concentration $\mathrm{C}$ versus $\mathrm{y}$ for $\mathrm{Re}=1, S c=0.22, m=10$ , $\operatorname{Pr}=0.71, t=1, \delta=0.5, M=10, G r=2, G m=1, Q=1, F=1$ $d_{1}=0.5, d_{2}=0.5, h=1, K=2 . S=1,2,3,4,5$

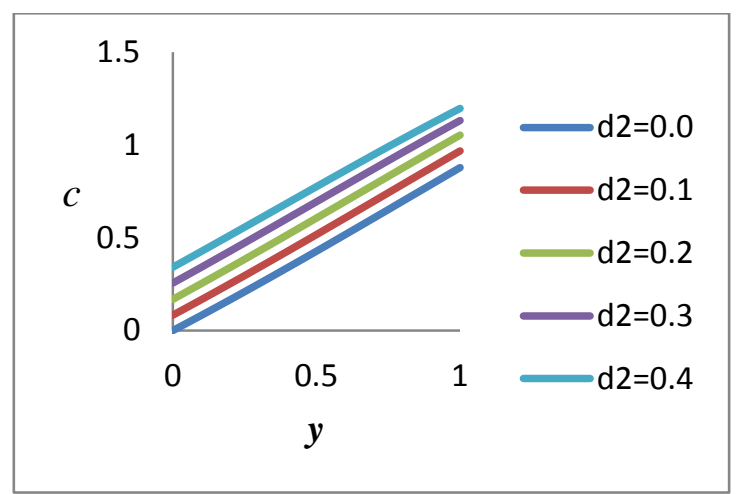

Figure 15: Concentration $\mathrm{C}$ versus y for $\mathrm{Re}=1, S c=0.22, S=1$, $\operatorname{Pr}=0.71, t=1, \delta=0.5, M=10, G r=2, G m=1, Q=1, F=1$ $d_{1}=0.5, m=10, h=1, K=2 ., d 2=0,0.1,0.2,0.3,0.4$

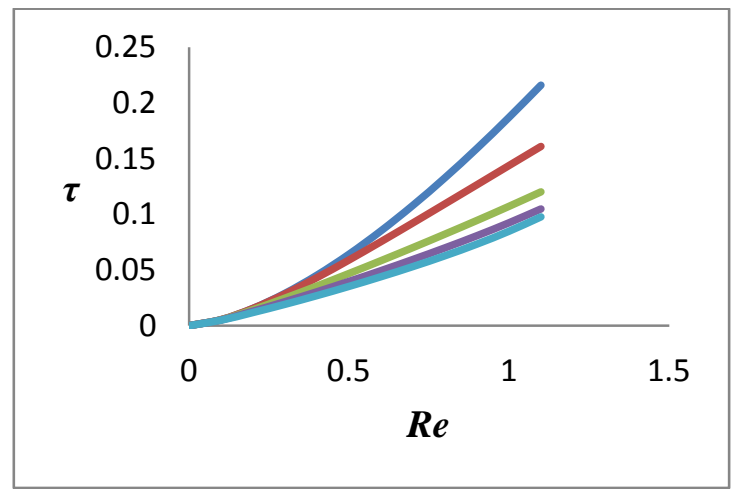

Figure 17: Skin friction $\tau$ versus $\operatorname{Re}$ for $m=10, S c=0.22, S=1$, $\operatorname{Pr}=0.71, t=1, \delta=0.5, M=10, G r=2, G m=1, F=1$, $d_{1}=0.5, d_{2}=0.5, h=1, K=2, Q=1,2,3,4,5$ at $\mathrm{y}=0$

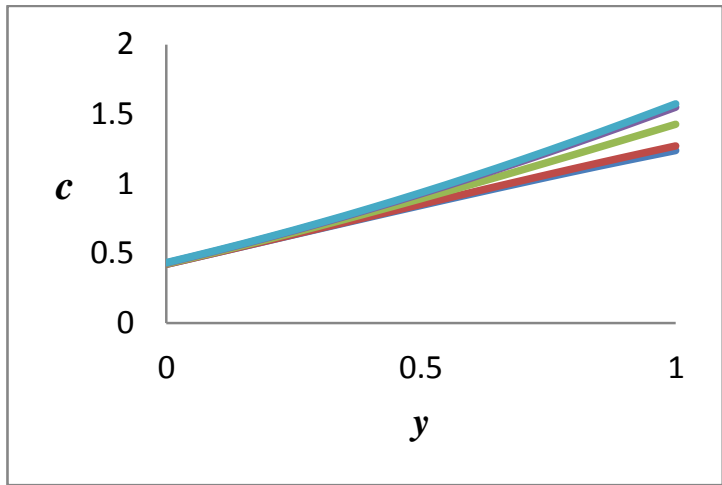

Figure 14: Concentration $\mathrm{C}$ versus y for $\mathrm{Re}=1, m=10, S=1$, $\operatorname{Pr}=0.71, t=1, \delta=0.5, M=10, G r=2, G m=1, Q=1, F=1$ $d_{1}=0.5, d_{2}=0.5, h=1, K=2, S c=0.22,0.3,0.6,0.78,0.81$

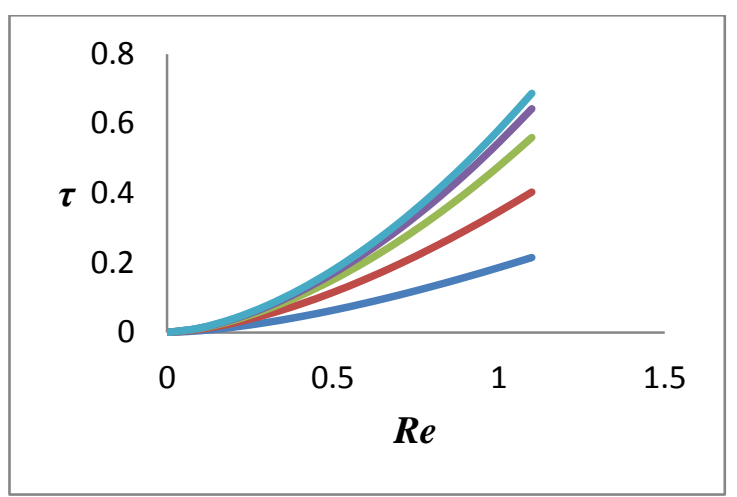

Figure 16: Skin friction $\tau$ versus Re for $S c=0.22, S=1$, $\operatorname{Pr}=0.71, t=1, \delta=0.5, M=10, G r=2, G m=1, Q=1, F=1$, $d_{1}=0.5, d_{2}=0.5, h=1, K=2 m=1,3,5,7,9$ at $\mathrm{y}=0$.

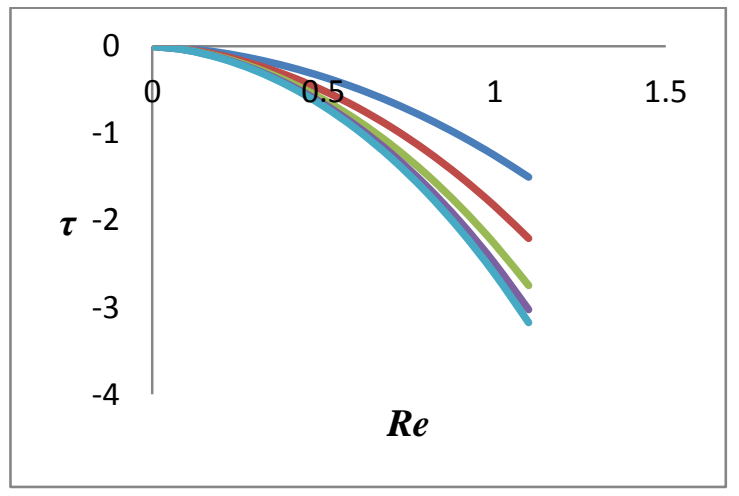

Figure 18: Skin friction $\tau$ versus $\operatorname{Re}$ for $S c=0.22, S=1$, $\operatorname{Pr}=0.71, t=1, \delta=0.5, M=10, G r=2, G m=1, Q=1, F=1$, $d_{1}=0.5, d_{2}=0.5, h=1, K=2, m=1,3,5,7,9$ at $\mathrm{y}=1$ 


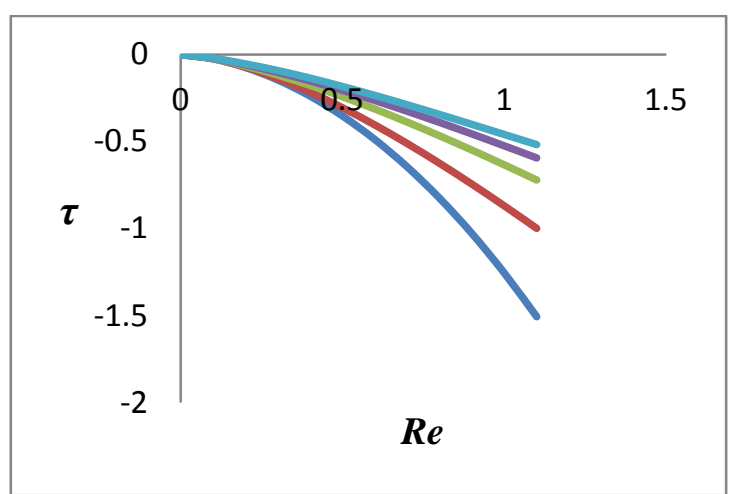

Figure 19: Skin friction $\tau$ versus Re for $m=10, S c=0.22, S=1$, $\operatorname{Pr}=0.71, t=1, \delta=0.5, M=10, G r=2, G m=1, F=1$, $d_{1}=0.5, d_{2}=0.5, h=1, K=2, Q=1,2,3,4,5$ at $\mathrm{y}=1$.

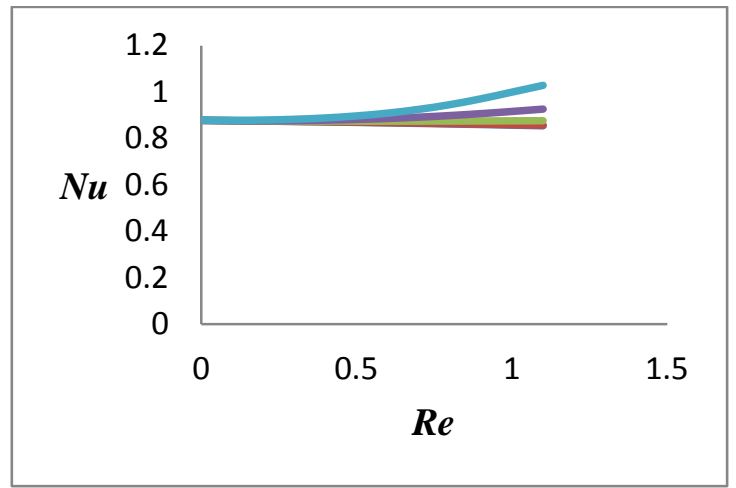

Figure 21: Nusselt Number Nu versus Re for $m=10, S c=0.22$, $Q=1 \operatorname{Pr}=0.71, t=1, \delta=0.5, M=10, G r=2, G m=1, F=1$, $d_{1}=0.5, d_{2}=0.5, h=1, K=2 \quad S=0.1,0.5,0.9,1.3,1.7$ at $\mathrm{y}=0$.

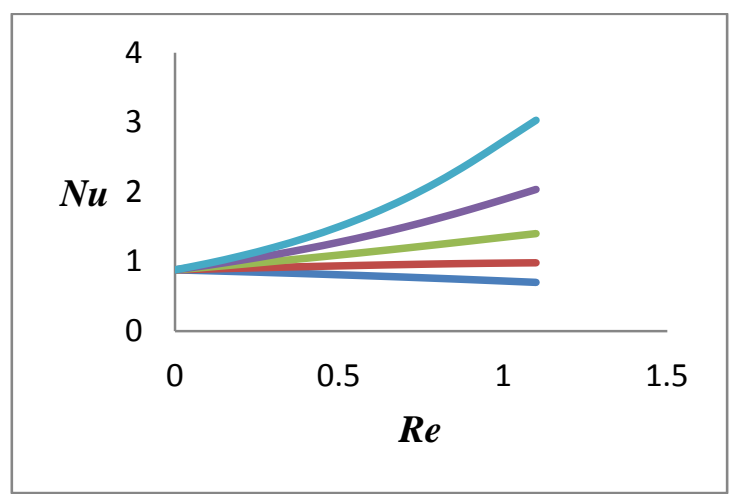

Figure 23: Nusselt Number Nu versus Re for $m=10, S c=0.22$, $Q=1 \operatorname{Pr}=0.71, t=1, \delta=0.5, M=10, G r=2, G m=1, F=1$, $d_{1}=0.5, d_{2}=0.5, h=1, K=2, S=0.1,0.5,0.9,1.3,1.7$ at $\mathrm{y}=1$

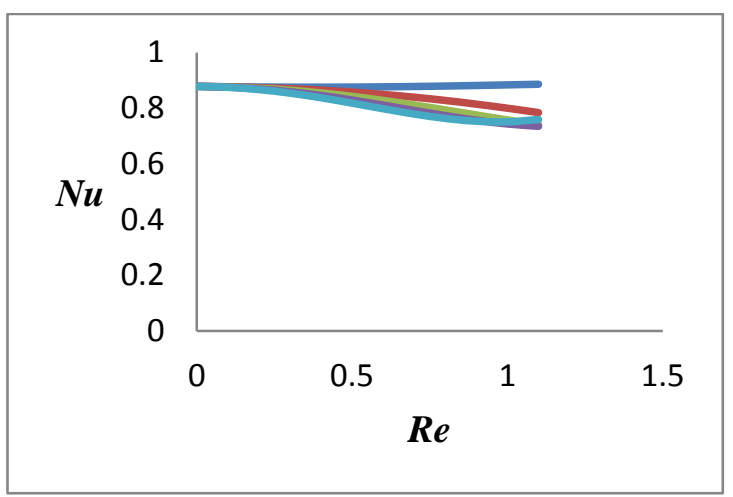

Figure 20: Nusselt Number Nu versus Re for $m=10, S c=0.22$, $S=1, \operatorname{Pr}=0.71, t=1, \delta=0.5, M=10, G r=2, G m=1, F=1$ $d_{1}=0.5, d_{2}=0.5, h=1, K=2 Q=1,2,3,4,5$ at $\mathrm{y}=0$.

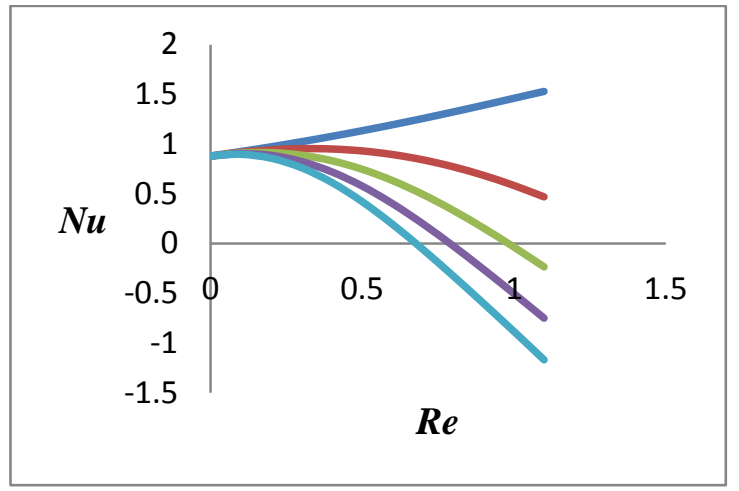

Figure 22: Nusselt Number Nu versus Re for $m=10, S c=0.22$, $S=1 \operatorname{Pr}=0.71, t=1, \delta=0.5, M=10, G r=2, G m=1, F=1$, $d_{1}=0.5, d_{2}=0.5, h=1, K=2, Q=1,2,3,4,5$ at $\mathrm{y}=1$.

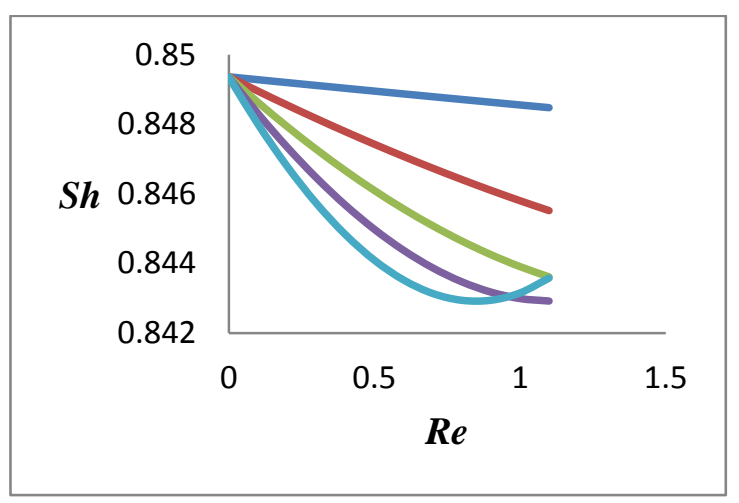

Figure 24: Sherwood Number Sh versus Re for $m=10, S c=0.22$ , $Q=1, \operatorname{Pr}=0.71, t=1, \delta=0.5, M=10, G r=2, G m=1, F=1$ $d_{1}=0.5, d_{2}=0.5, h=1, K=2, S=0.1,0.5,0.9,1.3,1.7$ at $\mathrm{y}=0$. 


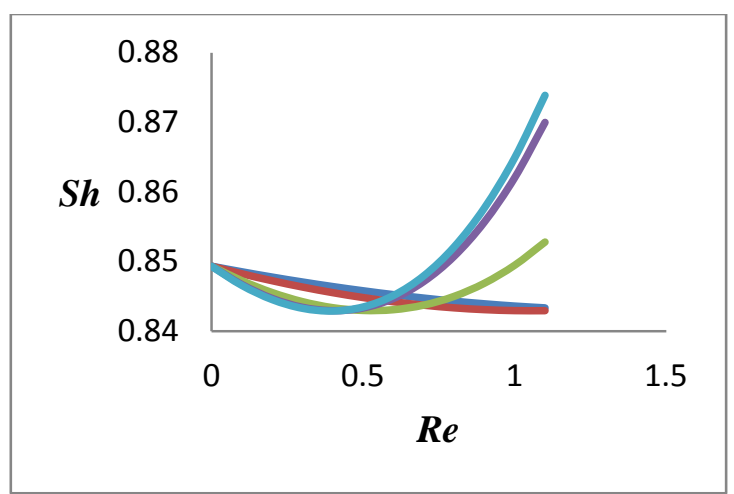

Figure 25: Sherwood Number Sh versus Re for $m=10, Q=1$ $\operatorname{Pr}=0.71, t=1, \delta=0.5, M=10, G r=2, G m=1, S=1, F=1$ $d_{1}=0.5, d_{2}=0.5, h=1, K=2, S c=0.22,0.3,0.68,0.78,0.81$ at $\mathrm{y}=0$.

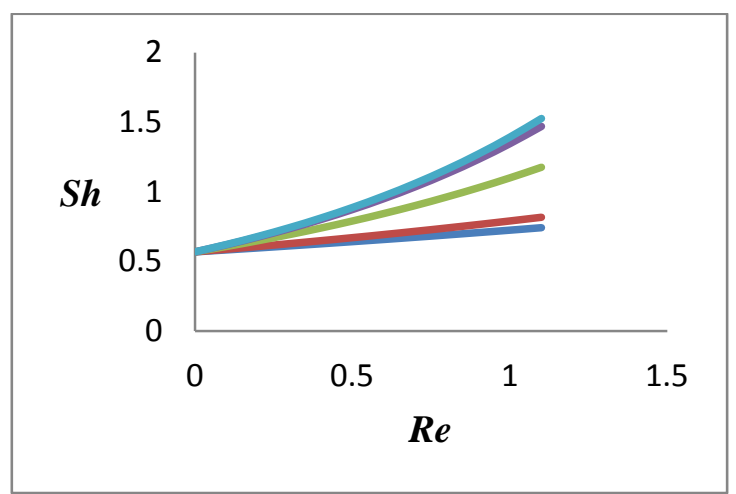

Figure 27: Sherwood Number Sh versus Re for $m=10, Q=1$ $\operatorname{Pr}=0.71, t=1, \delta=0.5, M=10, G r=2, G m=1, S=1, F=1$ $d_{1}=0.5, d_{2}=0.5, h=1, K=2, S c=0.22,0.3,0.68,0.78,0.81$ at $\mathrm{y}=1$.

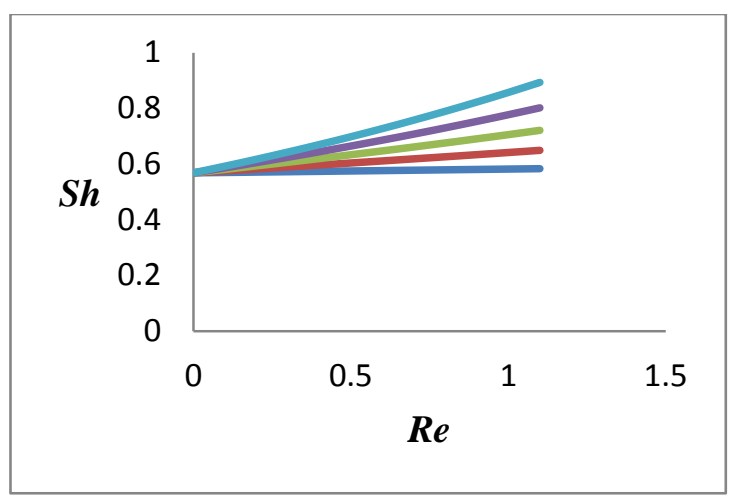

Figure 26: Sherwood Number $S h$ versus Re for $m=10, S c=0.22$, $Q=1, \operatorname{Pr}=0.71, t=1, \delta=0.5, M=10, G r=2, G m=1, F=1$ $d_{1}=0.5, d_{2}=0.5, h=1, K=2, S=0.1,0.5,0.9,1.3,1.7$ at $\mathrm{y}=1$.

\section{REFERENCES}

[1] Ahmed. N, Kalita. H and Barua. D. P., 2010, "Unsteady MHD free convective flow past a vertical porous plate immersed in a porous medium with Hall current, thermal diffusion and heat source”, Int. J. of Engg., Sci. and Tech., Vol. 2, no. 6, pp. 59-74.

[2] Anjali Devi . S. P. and Uma Devi. R, 2012, "Effects of thermal radiation on hydromagnetic flow due to a porous rotating disk with Hall effect", Journal of Applied Fluid Mechanics, Vol. 5, no. 2, pp. 1-7.

[3] Chand. K, Singh. K. D. and Kumar. S, 2012, "Hall effect on radiating and chemically reacting MHD oscillatory flow in a rotating porous vertical channel in slip flow regime", Adv. in Appl. Sci. Res., Vol. 3, no. 4, pp. 24242437.

[4] Cogley. A. C., Vincent. W. G. and Giles. S. E, 1968, "Differential approximation to radiative heat transfer in a non-grey gas near equilibrium", AIAA J, Vol. 6, no. 3, pp. 551-553.

[5] Kai-Long Hsiao,2016," Combined Electrical MHD Heat Transfer Thermal Extrusion System Using Maxwell Fluid with Radiative and Viscous Dissipation Effects", Applied Thermal Engineering.

[6] Kai-Long Hsiao, 2016, "Numerical solution for Ohmic Soret-Dufour Heat and Mass Mixed Convection of Viscoelastic Fluid over a Stretching Sheet with Multimedia Physical Features”, Journal of Aerospace Engineering.

[7] Raja Sekhar. K, Ramana Reddy. G. V and Prasad. B. D. C. N, 2012, "Chemically reacting on MHD oscillatory slip flow in a planer channel with varying temperature and concentration", Advances in Applied Science Research, Vol. 3, no. 5, pp. 2652-2659.

[8] Rakesh Kumar and Khem Chand, 2011,"Effect of slip conditions and hall current on unsteady MHD flow of a viscoelastic fluid past an infinite vertical porous plate through porous medium", Int. J. of Engg. Sci. and Tech. Vol. 3, no. 4, pp. 3124-3133. 
[9] Singh. K. D. and Kumar. R., 2010, “An exact solution of an oscillatory MHD flow through a porous medium bounded by rotating porous channel in the presence of hall current", Int. J. of Appl. Math and Mech., Vol. 6, no. 13, pp. 28-40.

[10] Sivaraj. R and Rushi Kumar. B., 2011, "Chemically reacting unsteady MHD oscillatory slip flow in a planar channel with varying concentration”, Int. J. of Math. and Sci. Computing, Vol. 1, no. 1, pp. 35-42. 